\title{
Comparison between C-arm cone beam computed tomography and interventional angiography in transarterial chemoembolization of hepatocellular carcinoma
}

\author{
Ibraheim Ahmed Diab', Shaimaa Abdel-hamid Hassanein ${ }^{2^{*}}$ (10) and Hala Hafez Mohamed²
}

\begin{abstract}
Background: Hepatocellular carcinoma (HCC) is the most common primary hepatic malignancy of adults. One of the established treatment procedures performed worldwide for HCC is transcatheter arterial chemoembolization (TACE). By using conventional angiography in TACE, we can detect and identify the vascular anatomy of the liver through obtaining 2D images. Recently C-arm cone beam computed tomography (CBCT) is introduced for obtaining cross-sectional and three-dimensional (3D) images for better visualization of small tumors and their feeding arteries.

Results: The number of detected focal lesions by angiography was 51 compared to 87 focal lesion detected by CBCT; of those, 45 and 77 were active lesions by both procedures respectively. For lesions, less than $1 \mathrm{~cm} C B C T$ detected 23 lesions while angiography detected only one lesion. Angiography detected 87 feeding arterial branch while cone beam CT-HA detected 130 branches to the same number of target lesion. Feeder tractability and confidence were better by CBCT.
\end{abstract}

Conclusion: $C B C T$ is superior to angiography in tumor detectability, detection of lesions less than $1 \mathrm{~cm}$, feeder detection, and feeder traction; however, conventional angiography and DSA are irreplaceable. Thus, combination of CBCT with angiography during TACE produces better results and less complication.

Keywords: Cone beam computed tomography, Hepatic angiography, Digital subtraction angiography, Hepatocellular carcinoma

\section{Background}

Hepatocellular carcinoma (HCC) is the most common primary hepatic malignancy of adults. It is the sixth most common cancer worldwide [1]. In Egypt, liver cancer forms $11.75 \%$ of the malignancies of all the digestive organs and $1.68 \%$ of the total malignancies. HCC constitutes $70.48 \%$ of all liver tumors among Egyptians [2].

HCC has a multitude of etiological risk factors, with hepatotropic viruses such as HBV, HCV, and hepatitis D

\footnotetext{
* Correspondence: shaimaahamid@hotmail.com; shaimaa.abd.alhameed66@med.menofia.edu.eg

${ }^{2}$ Radiology Department, Faculty of Medicine, Menoufia University, Yassin

Abdel-Ghafar Street, Shebin El-Kom, Menoufia, Egypt

Full list of author information is available at the end of the article
}

virus (HDV) showing a strong association with development of HCC; thus, the worldwide distribution of HCC mirrors the distributions of such viral infections [3], and since the prevalence of hepatitis $\mathrm{C}$ virus (HCV) infection in Egypt is the highest in the world [4], more cases of $\mathrm{HCC}$ are expected to be present.

One of the established HCC treatments, along with other treatments such as surgical resection and percutaneous ablation, is transcatheter arterial chemoembolization (TACE). With the recent new technologies, TACE treatment outcomes, namely the local suppression rate and survival rate, are improved compared with previous results [5-7]. 
Of these technologies is the use of $\mathrm{C}$-arm cone beam computed tomography (CBCT) using a flat-panel detector (FPD) which allowed obtaining cross-sectional and three-dimensional (3D) images during TACE unlike the two dimensional (2D) images of the vascular anatomy produced by the conventional angiography [8-11] that provided additional crucial information, such as visualization of small tumors and their feeding arteries, non-hepatic arteries, and possibly extrahepatic collateral arteries [8].

The aim of our study was to compare both modalities in cases of HCC undergoing TACE at our institution.

\section{Methods}

A prospective study was carried out on patients diagnosed with HCC by imaging and laboratory criteria, referred by the National Liver Institute oncology committee to the radiology department at the National Liver Institute to do TACE as the recommended treatment during the period from October 2017 to August 2018.

The study protocol was approved by the Medical Ethical Committee of Faculty of Medicine-Menoufia University. An informed consent was taken from each patient included in the study. All the patients underwent TACE using conventional angiography and $\mathrm{CBCT}$ techniques.

The inclusion criteria were HCC patients with wellpreserved liver function who refused the surgery or who were not suitable for resection, liver transplantation, or percutaneous ablation.

Those with advanced liver disease (Child-Pugh's C) and advanced tumoral disease (including vascular invasion, extrahepatic spread, and tumor $>50 \%$ of the whole liver) were excluded from the study. Renal insufficiency was also a contraindication for hepatic embolization procedures.

The included patients underwent thorough history taking and clinical assessment review of the laboratory studies performed (including complete blood count, liver function tests, renal function tests, and bleeding profile). Review of previous imaging studies such as abdominal ultrasound and abdominal triphasic CT study was performed prior to the procedure.

Pre-procedural preparation included the insertion of an 18-g cannula at the antecubital vein, fluid administration ( $500 \mathrm{cc}$ of normal saline intravenously), sedation (using midazolam $1 \mathrm{mg}$ IV), removal of any metals or clothes, and sterilization of the puncture site with povidone iodine.

The patients were then transformed to the angiography suite where the chemoembolization procedure was performed using angiography machine of (ALLURA XPER FD 20, Philips, Holland). After infiltration of local analgesic, femoral artery puncture was performed using the Seldinger technique to gain access to the common femoral artery.

A 5-Fr vascular sheath was placed into the common femoral artery over a 0.035-in. Guidewire (Terumo). Under fluoroscopic guidance, a 5-Fr Cobra catheter was advanced into the aorta. Angiographic studies of the superior mesenteric artery (SMA), celiac trunk, and common hepatic artery were performed to identify all of the vessels feeding the HCC nodule. In three patients, selective angiography of the phrenic and intercostal arterial branches was required. The arterial branches feeding the tumor were selectively cannulated by microcatheters to proceed with TACE and to ensure better preservation of the surrounding non-tumoral liver tissue.

Each angiography run was taken through injection of $10 \mathrm{~mm}$ of iodinated contrast media (Ultravest 300, Bayer Inc., Ontario, Canada) through the catheter or microcatheter. Images obtained from each angiography run were processed, and digital subtraction was performed by our device which is optimized to process the images automatically. Images were reviewed by the operator who determined the feeding branches and active lesions according to it.

During the procedure, at least two cone beam CT runs were taken in each patient, one from the main hepatic artery pre-embolization and the other post-embolization. Each run composed of two phases (arterial and portal); the arterial phase was taken through manual injection of $10 \mathrm{~mm}$ of non-diluted iodinated contrast media (Ultravest 300 , Bayer Inc., Ontario, Canada) at constant rate through the catheter or microcatheter and obtained images after $2 \mathrm{~s}$ from the start of injection by using the fast HD protocol in the device. The images were obtained in about 7-s duration where the patients were instructed to breath hold. After $20 \mathrm{~s}$ from the end of the arterial phase, another phase (portal) was taken using the same (fast HD) protocol. The images were processed automatically by the device producing the CT images which was reviewed by the operator.

Injection of $10 \mathrm{~cm}$ of lipiodol (iodized oil; Guerbet laboratories, Roissy, France) was done for each patient after determination of the target lesion and its feeding branches through angiography and CBCT.

\section{Post-procedure care}

Following the procedure, good hydration with $500 \mathrm{ml}$ normal saline was performed. Observation of the vital signs (particularly peripheral pulses were recorded periodically every $2 \mathrm{~h}$ for $24 \mathrm{~h}$ ) and puncture site (for at least $6 \mathrm{~h}$ ) was done and analgesics and antiemetic drugs were administered on need. Most of our patients were discharged after $24 \mathrm{~h}$ without any complications. 


\section{Image interpretation}

Images were transferred and stored on a picture archiving and communication system (IntelliSpace PACS radiology 4.4, Philips, USA), and an experienced interventional radiologist who was unaware of our study and its aim reviewed both angiography and cone beam CT images using Philips IntelliSpace Portal application (IntelliSpace Portal v.6, Philips, Netherlands) on BARCO medical monitors. He used both quantitative and qualitative analysis of the images' maximal intensity projection (MIP); multi-planar reconstruction (MPR) and volume rendering technique (VRT) reconstructions were done to the CBCT images; and digital subtraction angiography (DSA) was done to the conventional angiography images. All results obtained were compared to the results recorded by the operating intervention radiologist.

\section{Quantitative image analysis}

Images of both cone beam CT and angiography were analyzed regarding the number of focal lesions detected, size of smallest focal lesion, number of lesions less than $1 \mathrm{~cm}$, and the feeding branches and their order to each lesion.

\section{Qualitative image analysis}

The image quality was subjectively determined on a scale from 0 to 3 where 3 is excellent image quality, 2 is good image quality, 1 is fair image quality, and 0 is bad image quality. Artifacts also were subjectively determined on a scale from 0 to 3 where 0 refers to no artifacts, 1 refers to mild artifacts, 2 refers to moderate artifacts, and 3 refers to severe artifacts.

\section{Statistical analysis}

Data were analyzed using IBM Statistical Package for the Social Sciences (SPSS) version 20 for personal computers. Quantitative data were expressed as mean \pm standard deviation (SD). Qualitative data were expressed as number and percentage (no and \%) where chi-square test $\left(\chi^{2}\right)$ was used to compare between the two groups. $P$ value $<0.05$ was considered as statistically significant.

\section{Results}

Twenty-five patients were included in our study. They were 21 male $(84.0 \%)$ and 4 females $(16.0 \%)$ which reflects higher incidence of $\mathrm{HCC}$ in males more than females. Patients' ages ranged from 44 to 67 years old with a mean age of $56.08 \pm 6.35$ years.
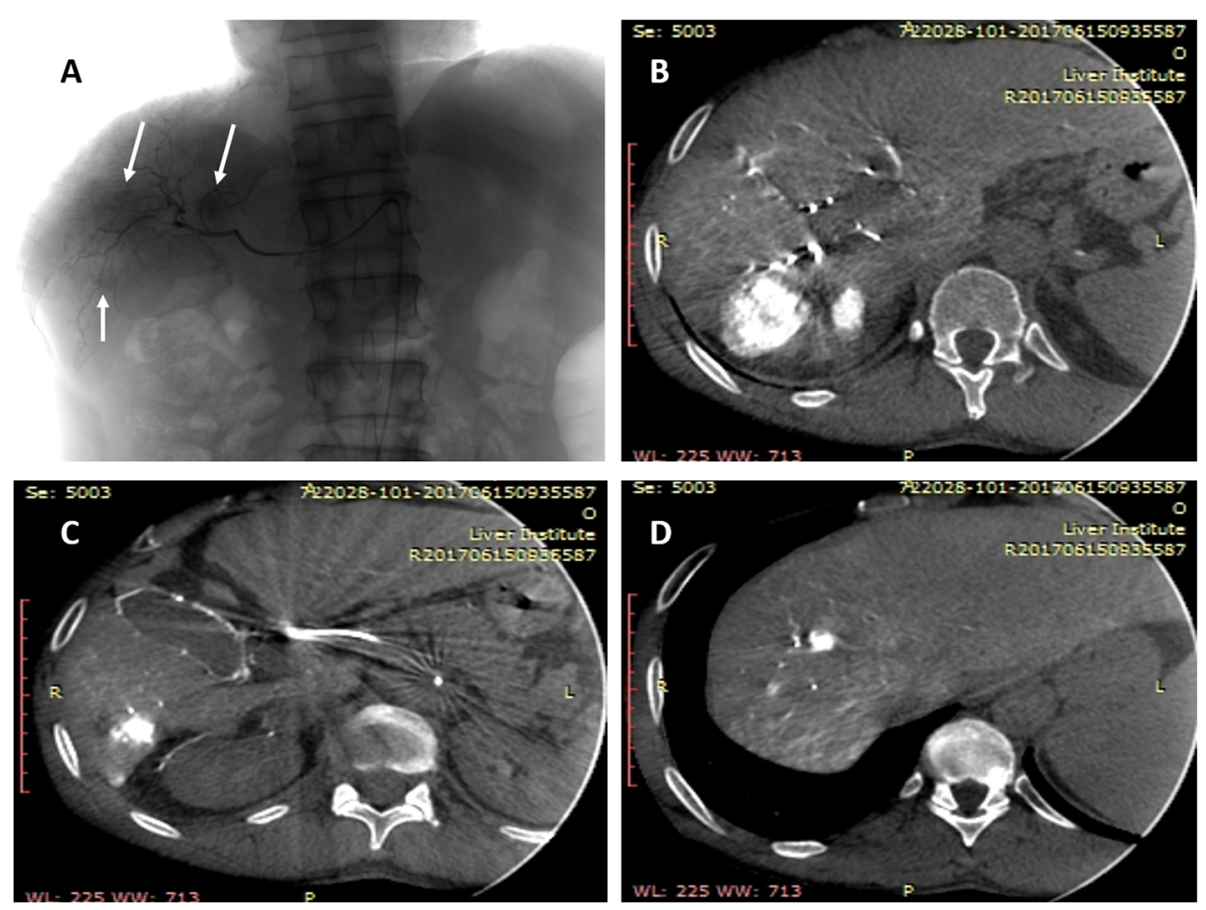

Fig. 1 A 51-year-old male patient with two focal lesions detected by ultrasound and confirmed to be HCC by imaging and laboratory criteria undergoing TACE as a treatment for HCC. a Non-selective angiography of the main right hepatic artery revealing the vascular anatomy of the right hepatic artery up to fourth-order branches and three enhanced focal lesions (arrows) and enhanced gall bladder wall. The size of the smallest lesion was $1.9 \mathrm{~cm}$. b, c Axial CBCT-HA revealing the vascular anatomy of the liver up to the fifth-order branches as well as the lesions that appeared in the angiography. The enhanced gall bladder wall also showed in $\mathbf{c}$. $\mathbf{d}$ Axial CBCT-HA at higher cuts revealing two additional smaller lesions. The smallest lesion was $0.8 \mathrm{~cm}$ 
Fifty-one focal lesions were detected by angiography of those only one was less than $1 \mathrm{~cm}$ while the number increased to 87 focal lesion detected by cone beam CT of those 23 lesions were less than $1 \mathrm{~cm}$ in maximal diameter (Fig. 1). The number of active focal lesions was 45 $(88.2 \%)$ by non-selective angiography compared to 77 (88.5\%) detected by cone beam CT.

Of those 45 active lesions, 43 were selected target lesions. Conventional angiography detected 87 feeding arterial branches to those lesions with a mean of $3.48 \pm$ 1.674 branch for each target lesion while CBCT detected larger number of branches (130 branches) to the same number of target lesions (43) with a mean of $5.18 \pm$ 2.264 branches for each target lesion.

In all the 25 patients, the main hepatic artery, hepatic artery proper, main left hepatic artery, and main right hepatic artery could be tracked by both $\mathrm{CBCT}$ and angiography. However, first-order branches were tracked in 24 patients by angiography and 25 patients by CBCT, second-order branches were tracked in 22 patients by angiography and 23 by CBCT, third-order branches were tracked in 12 patients by angiography and in 16 patients by $\mathrm{CBCT}$, and fourth-order branches were tracked in 4 patients by angiography and 13 patients by $\mathrm{CBCT}$, while the fifth-order branches were tracked in 6 cases by CBCT but could not be tracked in any case using angiography.

Regarding the confidence of the observer about the tumor feeding branches using CBCT (Fig. 2), observers had a good confidence in about $80 \%$ of the reviewed arteries and fair confidence in $15.3 \%$ of the reviewed feeders and only $4.7 \%$ of the reviewed were of poor confidence. On the other hand, the confidence was lower when observing the feeders of the same detected lesions by angiography, where the good confidence was $57.5 \%$, fair was $31 \%$, and poor was $11.5 \%$ of the reviewed feeders. And regarding to the tractability of the tumor feeding branches, $75.3 \%$ of the reviewed arteries detected by CBCT could be tracked good (at least tracked to the fourth-order branches), $18.5 \%$ tracked fair (at least tracked to second-order branches), and 6.1\% tracked poor (tracked maximally up to the first-order branches only).

We studied motion, lipiodol-induced, and catheterinduced artifacts within the images of both modalities. No induced artifact was highly significantly prevalent by angiography than CBCT $(P$ value $<0.001)$. Moreover, mild, moderate, or severe artifact induced by angiography was significantly lower than that induced by CBCT ( $P$ value $<0.05)$ (Table 1 , Fig. 3 ).

The prevalence of excellent image quality was significantly higher by angiography (64.8\%) than with CBCT $(7.5 \%)$ ( $P$ value $<0.001)$. On the other hand, bad, fair, and good image quality were significantly higher by $\mathrm{CBCT}$ (bad $5.7 \%$, fair $18.9 \%$, and good $67.9 \%$ ) than by angiography (bad $0.7 \%$, fair $6.2 \%$, and good $28.3 \%$ ) ( $P$ value $<0.05$ ).

The angiography showed better coverage of the whole liver in the same field of view (in $89.6 \%$ angiography acquisitions compared to $28.3 \%$ in $\mathrm{CBCT}$ ).

\section{Discussion}

One of the keys to successful TACE lies in seeing and reaching tumor lesions after accurate and
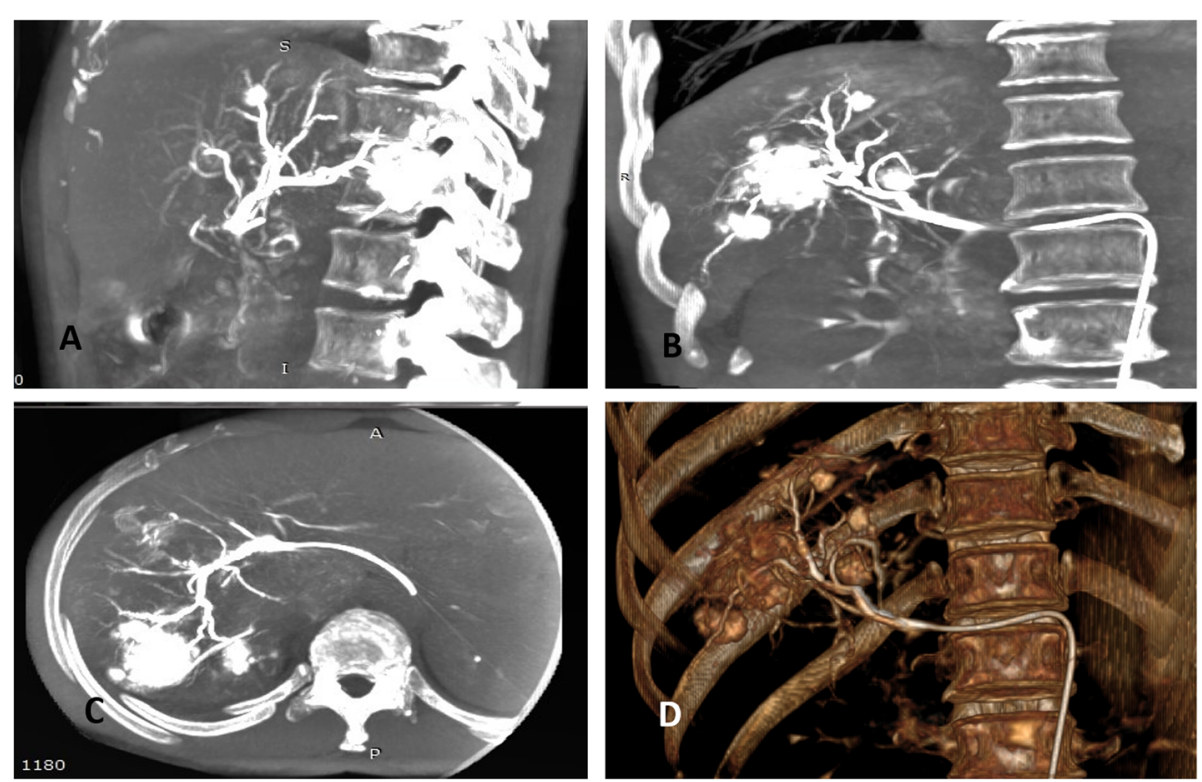

Fig. 2 Multiplanar reconstruction with the use of maximum intensity projection (MIP) of CBCT-HA in a case of multi-focal HCC (a sagittal, b coronal, and $\mathbf{c}$ axial) revealing the feeding arteries of the different lesions even the smallest ones. $\mathbf{d}$ 3D virtual reconstruction revealing the feeding arteries with good confidence even in the presence of bones 
Table 1 Degree of image artifacts due to motion, catheter, and lipiodol in each acquisition taken by angiography and CBCT

\begin{tabular}{|c|c|c|c|c|c|c|c|c|c|c|c|c|}
\hline \multirow{3}{*}{$\begin{array}{l}\text { Degree } \\
\text { of artifact }\end{array}$} & \multicolumn{4}{|c|}{ Motion artifact } & \multicolumn{4}{|c|}{ Lipiodol-induced artifact } & \multicolumn{4}{|c|}{ Catheter-induced artifact } \\
\hline & \multicolumn{2}{|c|}{ Angiography (no = 145) } & \multicolumn{2}{|c|}{$\mathrm{CBCT}(\mathrm{no}=106)$} & \multicolumn{2}{|c|}{ Angiography $(\mathrm{no}=145)$} & \multirow{2}{*}{\multicolumn{2}{|c|}{$\frac{\mathrm{CBCT}(\mathrm{no}=106)}{\text { No } \%}$}} & \multicolumn{2}{|c|}{ Angiography $(\mathrm{no}=145)$} & \multicolumn{2}{|c|}{$C B C T(n o=106)$} \\
\hline & No & $\%$ & No & $\%$ & No & $\%$ & & & No & $\%$ & No & $\%$ \\
\hline Non & 94 & 64.8 & 40 & 37.7 & 110 & 75.9 & 8 & 7.5 & 130 & 89.7 & 8 & 7.5 \\
\hline ( $P$ value) & \multicolumn{4}{|c|}{$(<0.001)$} & \multicolumn{4}{|c|}{$(<0.001)$} & \multicolumn{4}{|c|}{$(<0.0001)$} \\
\hline Mild & 41 & 28.3 & 40 & 37.7 & 35 & 24.1 & 82 & 77.4 & 15 & 10.3 & 80 & 75.5 \\
\hline ( $P$ value) & \multicolumn{4}{|c|}{$(0.11)$} & \multicolumn{4}{|c|}{$(<0.001)$} & \multicolumn{4}{|c|}{$(<0.001)$} \\
\hline Moderate & 9 & 6.2 & 20 & 18.9 & 0 & 0 & 8 & 7.5 & 0 & 0 & 16 & 15.1 \\
\hline ( $P$ value) & \multicolumn{4}{|c|}{$(<0.001)$} & \multicolumn{4}{|c|}{$(<0.001)$} & \multicolumn{4}{|c|}{$(<0.001)$} \\
\hline Severe & 1 & 0.7 & 6 & 5.7 & 0 & 0 & 8 & 7.5 & 0 & 0 & 4 & 3.8 \\
\hline ( $P$ value) & \multicolumn{4}{|c|}{$(0.02)$} & \multicolumn{4}{|c|}{$(<0.001)$} & \multicolumn{4}{|c|}{$(<0.02)$} \\
\hline
\end{tabular}

comprehensive assessment of all the feeding arteries. Conventionally, operators rely on fluoroscopy and digital subtraction angiography (DSA). However, the intrinsic two-dimensional nature of DSA and its low contrast resolution limit tumor detection, resulting in a recent adoption of intraprocedural cone beam computed tomography (CBCT) for its axial and three-dimensional (3D) volumetric visualization, as well as superior soft tissue sensitivity [8-11].
To our knowledge, no study comparing the two modalities has been conducted in such an endemic area as Egypt.

Our included study population were 21 males $(84.0 \%)$ and 4 females $(16.0 \%)$ that emphasized the more prevalence of $\mathrm{HCC}$ in males going with the results of Liu et al. [12] who showed that HCC was more prevalent in males than females but with no exact clear etiology of that difference except for the hypothesis of the
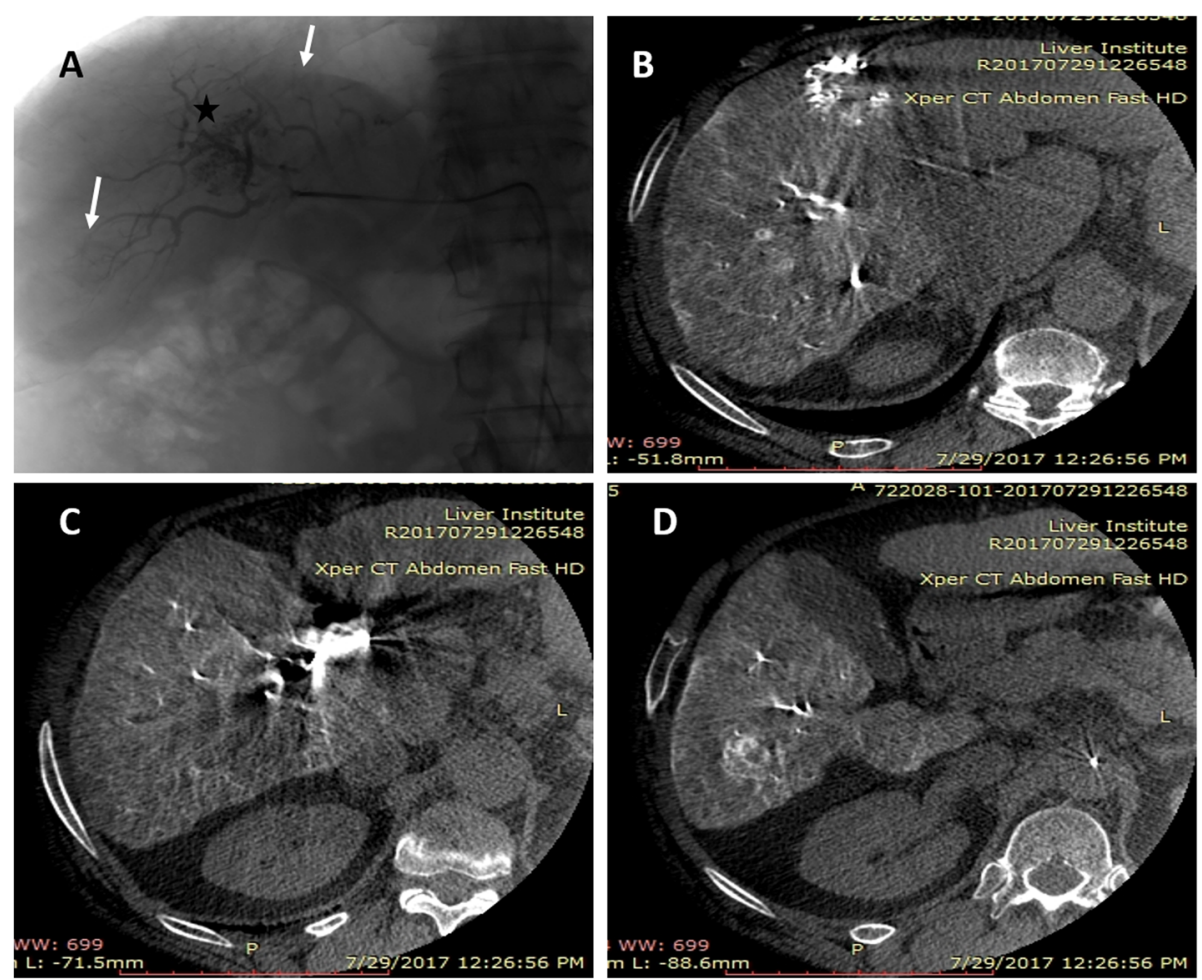

Fig. 3 A 67-year-old male patient coming for a second TACE session for a newly developed HCC focus. a Non-selective angiography of the main right hepatic artery revealing the vascular anatomy of the right hepatic artery up to fifth-order branches as well as the hyper-dense concentrates of the previously managed hepatic focal lesion (asterisk) and de novo two enhanced focal lesions (arrows). No catheter or lipiodol-induced artifacts could be detected. $\mathbf{b}$ - $\mathbf{d}$ Images of CBCT of the same patient showing mild lipiodol streak artifacts of the previously injected lesion (b), moderate catheter-induced artifacts (c), and mild motion artifacts (d) appearing as double contour of the vessels; $\mathbf{d}$ also shows the de novo focal lesion 
protective effect of female sex hormones against HCC development as most of the detected HCC were in postmenopausal females with higher mean age of females with HCC than that of males. This was also the case in our study as the mean age of the male group was $55.67 \pm 6.814$ years while that of the female group was $58.25 \pm 2.363$ years. Holah et al. [13] and Ziada et al. [14] reached the same conclusion that HCC was more prevalent in males than females.

The whole study group mean age was $56.08 \pm 6.350$ years. Similar results were reported by Ziada et al. [14], Mohamed et al. [15], and Tangkijvanich et al. [16] who reported a mean age of $57.5,56$, and 52.6 years respectively for HCC in high risk areas.

CBCT showed higher tumor detectability than angiography; the number of focal lesions detected by CBCT in our study was 87 while only 51 focal lesions were detected by angiography (Fig. 4). Similar results were reported by Yao et al. [17] who studied 43 HCC patients in which CBCT detected 99 lesions while angiography detected only 82 lesions. That emphasized the higher tumor detectability of dual-phase CBCTs than DSA. The mean number of lesions detected in each patient in our study by CBCT was 3.48 lesions while in the study by Yao et al. [17], it was 2.3 lesions; this could be attributed to the fact that our study was conducted in a high-risk region (Menoufia, Egypt) in which there is a high incidence of HCC.

Similar results about the tumor detectability where reported by Zheng et al. [18], Kakeda et al. [19], and Wang et al. [20] where cone beam CT outperformed angiography (DSA) in all the three studies.

We were able to detect 23 lesions less than $1 \mathrm{~cm}$ in diameter by dual-phase cone beam CT while only 1 lesion was detected by angiography. Similar results were reported by Lee et al. [21] in which all tumors less than $1 \mathrm{~cm}$ were detected by CBCT (23 tumors). Lee et al. [21] accounted the high detectability of CBCT to the small lesions less than $1 \mathrm{~cm}$ to the small focal spot and the high spatial resolution of CBCT more than the multidetector $\mathrm{CT}$ and the conventional angiography.

Similar results about the detection of small focal lesions less than $1 \mathrm{~cm}$ were reported in the meta-analysis done by Pung et al. [22] who reported that tumors more than 30 $\mathrm{mm}$ were easier to be detected by cone beam CT; however, the advantage of the high-contrast, three-dimensional images remained for tumors less than $10 \mathrm{~mm}$ and the tumors that could not be detected by angiography (Fig. 5).

In contrast, Kakeda et al. [19] reported that CBCT imaging did not detect tumors but detected the feeding
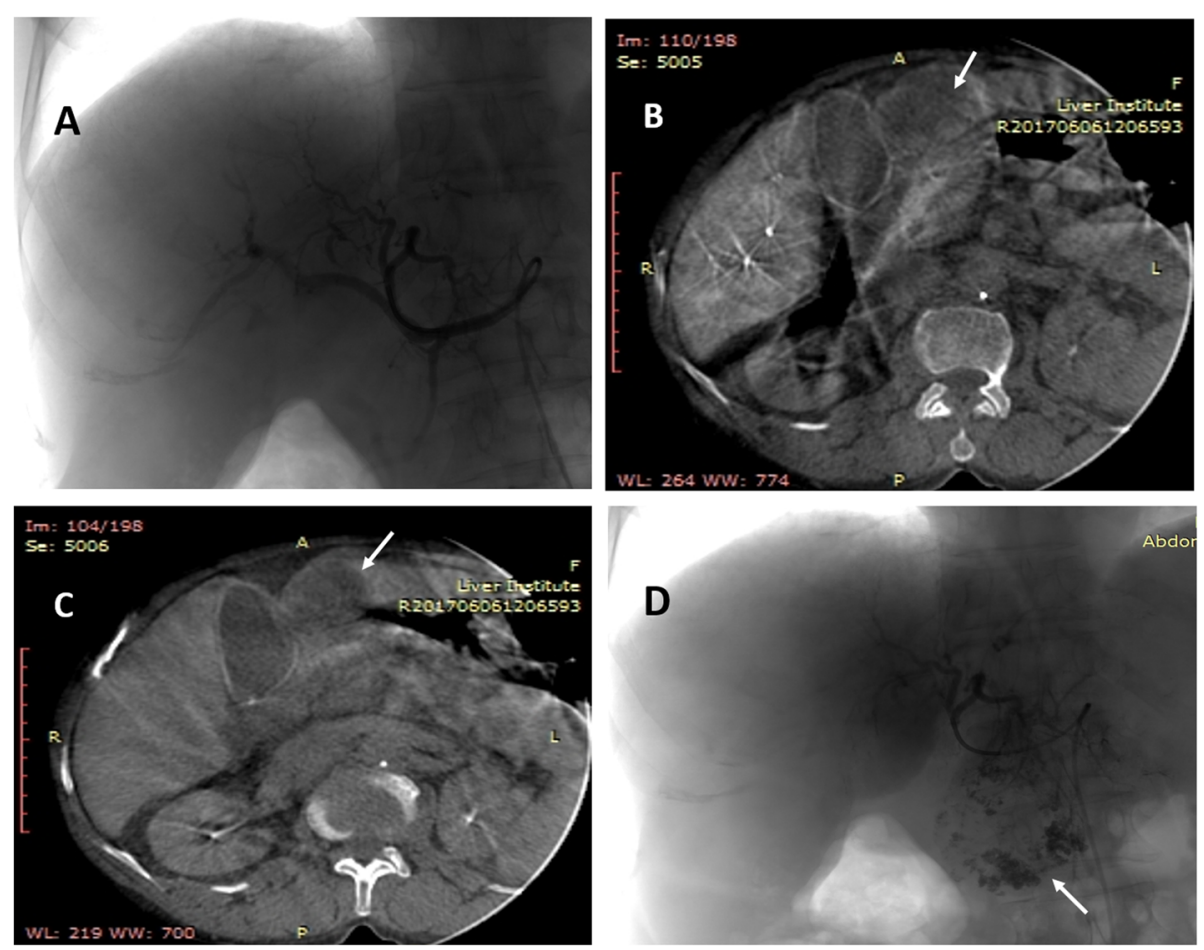

Fig. 4 A 68-year-old female patient diagnosed to have left lobe HCC focal lesions by laboratory and imaging criteria referred to do TACE. a Nonselective angiography of the main left hepatic artery revealing the vascular anatomy of the left hepatic artery with no detected focal lesions. $\mathbf{b}$ Axial CBCT-HA revealing left lobe mild peripherally enhancing focal lesion (arrow). c Axial CBCT-PV revealing wash out of contrast from the left lobe focal lesion (arrow). $\mathbf{d}$ Non-selective angiography of the main left hepatic artery post-lipiodol injection revealing concentration of lipiodol in the left lobe lesion (arrow) which was not shown in the angiography before 
arteries. This is believed to be because their evaluation was based only on MIP and VR images, with small or weakly enhanced nodules not being revealed during three-dimensional reconstruction. In our study, evaluation was made using three-dimensional MPR images as well as MIP and VR images. Evaluation with MPR images is considered necessary for the identification of small nodules and nodules with weak enhancement. It is believed that CBCT imaging could become a useful tool for identification of such small nodules.

As regards the feeding arteries, conventional angiography detected 87 feeding arterial branches to 43 target lesions with a mean of $3.48 \pm 1.674$ branches for each target lesion. On the other hand, cone beam computed tomography-hepatic artery phase (CBCT-HA) detected 130 branches to the same number of target lesion with a mean of $5.18 \pm 2.264$ branches for each target lesion. Miyayama et al. [23] reported similar results as among 100 tumor-feeding vessels, 81 were identified with cone beam CT manual feeding branch detection and 38 with non-selective DSA. They compared both methods with multidetector CT revealing statistically significant higher detectability of tumors and tumor-feeding branches with MFD than with non-selective DSA (both $P<.001$ ). They were able to calculate the $P$ value as they compared both to multidetector $\mathrm{CT}$ results which were not available in our study.

Similar results about the detectability of feeding branches by cone beam CT and angiography were reported by Ushijima et al. [24] who reported that 53 feeding arteries were associated with the studied 39 HCC nodules. Among them, 21 arteries were identified by DSA imaging (angiography from the proximal hepatic artery); however, 26 additional feeding arteries were identified by combined application of DSA and CBCT imaging. Moreover, Minami et al. [25] also reported that the $\mathrm{CBCT}$ tracking navigation imaging detected 66 out of 73 feeding branches $(90.4 \%)$ while celiac trunk nonselective angiography detected only 37 out of 73 feeding branches (50\%). They used selective angiography to each lesion as a gold standard, but in our study, we only compared the number of feeders detected by each modality.

The meta-analysis done by Pung et al. [22] concluded that the overall detectability of the tumor feeding branches by CBCT is much better than that of conventional angiography whether selective, non-selective, or celiac trunk angiography. However, there are factors that negatively influenced the accuracy of cone beam CT which includes the small caliber of the feeding artery, poor patients' breath hold, adjacent arterio-portal shunts, or streak artifact from metal clips.
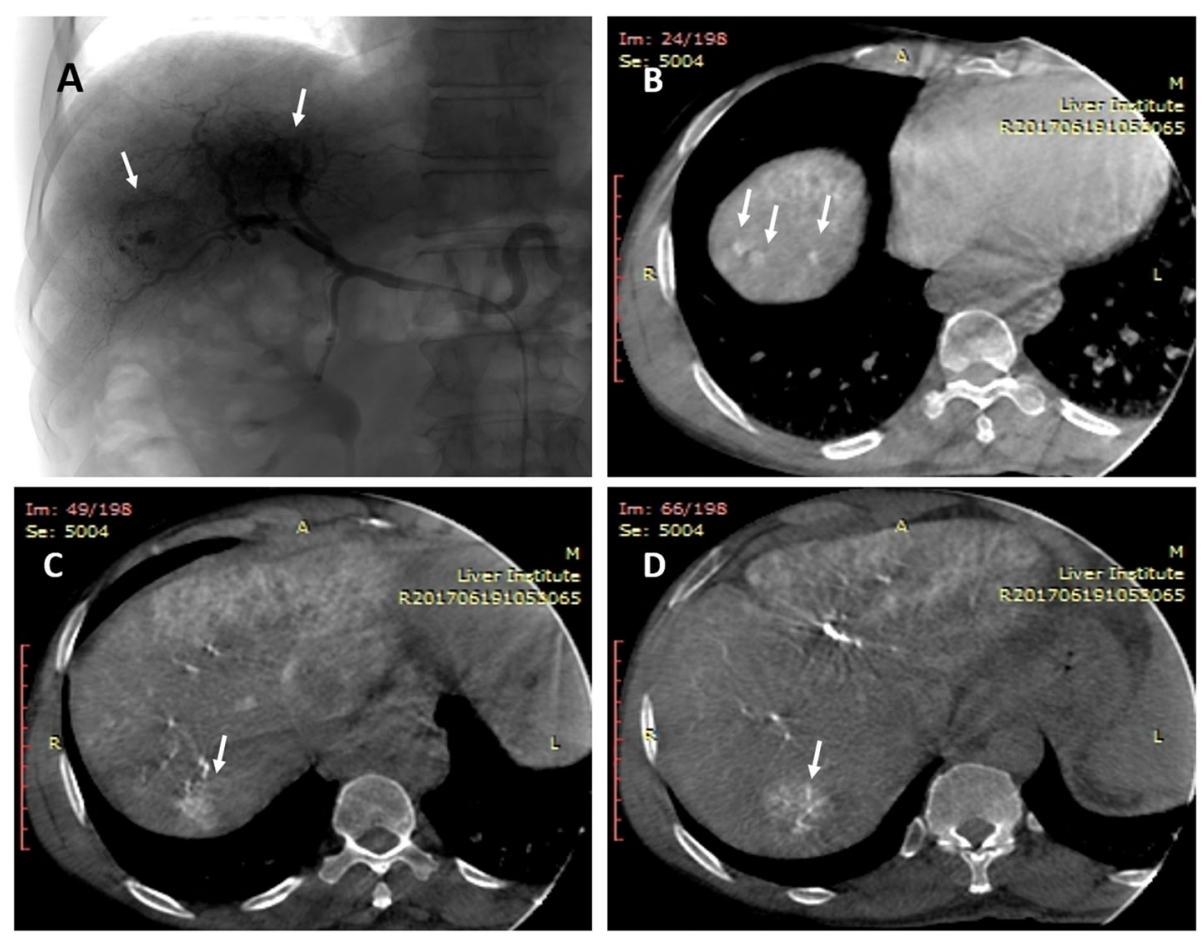

Fig. 5 A 66-year-old male patient diagnosed to have three HCC focal lesions by laboratory and imaging criteria referred to do TACE. a Nonselective angiography of the main right hepatic artery revealing the vascular anatomy of the right hepatic artery up to fourth-order branches as well as two enhanced focal lesions (arrows). b-d Axial CBCT-HA at different levels from superior to inferior demonstrating five focal lesions, three of them were not seen by angiography or any imaging modality, the size of the smallest one was $6 \mathrm{~mm}$ 
Our study reported that the confidence of tumor feeding branches was much better using $\mathrm{CBCT}$ than angiography; there was good confidence in $80 \%$ of the reviewed arteries, fair confidence in $15.3 \%$ of the reviewed feeders, and poor confidence in $4.7 \%$ of the reviewed feeders. But when observing the feeders of the same lesions detected by angiography, good confidence was in $57.5 \%$, fair in $31 \%$, and poor in $11.5 \%$ of the reviewed feeders. This is because of the 3D capability of CBCT over 2D angiography as the liver, specially its right and caudate lobes, show many overlapping anterior and posterior branches in the 2D images of the antero-posterior direction of angiography and DSA. These can be easily separated using the combination of the MPR, MIP, and 3D VR reconstructions of the CBCT images as we did in our study.

Similar results were reported by Ushijima et al. [24] who concluded that CBCT imaging was better in identifying feeding arteries associated with lesions located in the right hepatic lobe, as this lobe is deep anteroposteriorly and its anterior and posterior branches often overlap, leading to poor isolation in $2 \mathrm{D}$ images of the antero-posterior direction.

For the similar cause of the overlapping arteries and the better spatial resolution, Lee et al. [26] reported that CBCT was superior to angiography in tumor feeding branches tractability which is an important advantage when performing chemoembolization for HCC with CBCT hepatic arteriography as it enables the demonstration of subtle feeding arteries and provide a threedimensional roadmap. This also agrees with our results.

In spite of the superiority of CBCT in tumor detection and feeding vessels isolation, we observed that the images' quality is affected by motion, catheter-induced, and lipiodol-induced artifacts more than does conventional angiography and DSA. Our study reported that out of 145 angiography acquisition, 94 acquisitions had no motion, catheter-induced, or lipiodol-induced artifacts while out of 106 CBCT acquisitions, only 8 acquisitions had no artifacts.

Our study revealed that $6 \mathrm{CBCT}$ acquisitions were severely compromised by all types of artifacts and had bad image quality while only 1 angiography acquisition was severely compromised by all types of artifacts and had a bad image quality. That is attributed to the fact that CBCT acquisition requires more time (about $12 \mathrm{~s}$ ) of no motion, breath holding, and steady contrast injection rate while angiography takes only about 4 to $6 \mathrm{~s}$ and also CBCT has exaggerated beam hardening. Similar results were reported by Lee et al. [26].

The whole liver was covered during image acquisition in $89.6 \%$ of angiography acquisitions and $28.3 \%$ of CBCT acquisitions which demonstrates the superiority of angiography over CBCT in liver coverage in the same acquisition. Lee et al. [26] reported that in 29 of 100 cases, CBCT covered the whole liver while incomplete coverage was found in the remaining 71 cases. They noticed that the areas most often missed from the field of view of cone beam CT were the left lateral segments and the inferior portion of segment VI, and this was also observed in our study.

\section{Conclusion}

$\mathrm{CBCT}$ is a valuable addition in the TACE procedure for management of HCC since it overcomes angiography in tumor detectability, detection of lesions less than $1 \mathrm{~cm}$, feeder detection, and feeder traction; however, conventional angiography and DSA are still irreplaceable. Hence, a tailored plan for each patient using the combination of $\mathrm{CBCT}$ and angiography during TACE produces is recommended for better results and less complications.

\section{Abbreviations \\ CBCT-HA: Cone beam computed tomography-hepatic artery phase; \\ FPD: Flat-panel detector; HCC: Hepatocellular carcinoma; TACE: Transarterial chemoembolization}

\section{Acknowledgements \\ The authors acknowledge the participations of the technicians of the interventional unit at our institution.}

\section{Authors' contributions}

IAD gave idea and collected the patients' data and analyzed them. SAH revised the patients' imaging modalities postoperatively and wrote the paper with revision. $\mathrm{HH}$ put the study design and revised the results and the paper. They all approved the final version of the manuscript.

Funding

This study had no funding from any resource.

Availability of data and materials

The datasets used and/or analyzed during the current study are available from the corresponding author on reasonable request.

Ethics approval and consent to participate

This study was approved by the Research Ethics Committee of the Faculty of Medicine at Menoufia University in Egypt on 10 October 2017; reference number of approval: 19719RADIO.

All patients included in this study gave written informed consent to participate in this research.

Consent for publication

All patients included in this research gave written informed consent to publish the data contained within this study.

\section{Competing interests}

The authors declare that they have no competing interests.

\section{Author details}

${ }^{1}$ Radiology Department, National Liver Institute Menoufia University, Yassin Abdel-Ghafar Street, Shebin El-Kom, Menoufia, Egypt. ${ }^{2}$ Radiology Department, Faculty of Medicine, Menoufia University, Yassin Abdel-Ghafar Street, Shebin El-Kom, Menoufia, Egypt. 
Received: 18 June 2019 Accepted: 23 September 2019

Published online: 29 November 2019

\section{References}

1. Dai L, Ren P, Liu M, Imai H, Tan EM, Zhang JY (2014) Using immunomic approach to enhance tumor-associated autoantibody detection in diagnosis of hepatocellular carcinoma. Clin Immunol 152:127-139

2. Mokhtar N, Gouda I, Adel I (2007) Cancer pathology registry 2003-2004 and time trend analysis. In: Mokhtar N, Gouda I, Adel I (eds) Malignant digestive system tumors. Elsheraa Press, Cairo, pp 55-67

3. El-Serag HB (2011) Hepatocellular carcinoma. N Engl J Med 365:1118-1127

4. Blach S, Zeuzem S, Manns M et al (2016) Global prevalence and genotype distribution of hepatitis C virus infection in 2015: a modeling study. Lancet Gastroenterol Hepatol 2(3):161-176

5. Miraglia R, Pietrosi G, Maruzzelli L, Petridis I, Caruso S, Marrone G, Mamone G, Vizzini G, Luca A, Gridelli B (2007) Efficacy of transcatheter embolization/ chemoembolization (TAE/TACE) for the treatment of single hepatocellular carcinoma. World J Gastroenterol 13:2952-2955

6. Camma C, Schepis F, Orlando A, Albanese M, Shahied L, Trevisani F, Andreone P, Craxi A, Cottone M (2002) Transarterial chemoembolization for unresectable hepatocellular carcinoma: meta-analysis of randomized controlled trials. Radiology 224:47-54

7. Llovet JM, Bruix J (2003) Systematic review of randomized trials for unresectable hepatocellular carcinoma: chemoembolization improves survival. Hepatology 37:429-442

8. Floridi C, Radaelli A, Abi-Jaoudeh N et al (2014) C-arm cone-beam computed tomography in interventional oncology: technical aspects and clinical applications. Radiol Med 119:521-532. https://doi.org/10.1007/ s11547-014-0429-5

9. Tacher V, Radaelli A, Lin M et al (2015) How I do it: cone beam computed tomography during transarterial chemoembolization for liver cancer. Radiology 274:320-334. https://doi.org/10.1148/radiol.14131925

10. Iwazawa J, Ohue S, Hashimoto $\mathrm{N}$ et al (2013) Clinical utility and limitations of tumor-feeder detection software for liver cancer embolization. Eur J Radiol 82:1665-1671

11. Tacher V, Lin M, Bhagat N et al (2013) Dual-phase cone-beam computed tomography to see, reach, and treat hepatocellular carcinoma during drugeluting beads transarterial chemo-embolization. J Vis Exp 2:50795

12. Liu P, Xie S, Hu S et al (2017) Age-specific sex difference in the incidence of hepatocellular carcinoma in the United States. Oncotarget 8(40):68131-68137

13. Holah NS, El-Azab DS, Aiad HA, Sweed DM (2015) Hepatocellular carcinoma in Egypt: epidemiological and histopathological properties. Menoufia Med J 28(3):718-724

14. Ziada DH, El-Sadany S, Soliman H et al (2016) Prevalence of hepatocellular carcinoma in chronic hepatitis C patients in Mid Delta, Egypt: a single center study. J Egypt Natl Canc Inst 28(4):257-262

15. Mohamed NH, El-Zawahry HM, Mokhtar NM et al (2000) Review of epidemiologic and clinicopathologic features of 403 hepatocellular carcinoma (HCC) patients. J Egypt National Cancer Inst 12(2):87-93

16. Tangkijvanich $P$, Mahachai $V$, Suwangoo IP, Poovorawan Y (2004) Gender difference in clinicopathologic features and survival of patients with hepatocellular carcinoma. World J Gastroenterol 10(11):1547-1550

17. Yao $X$, Yan D, Jiang $X$, et al. Dual-phase cone-beam CT-based navigation imaging significantly enhances tumor detectability and aids superselective transarterial chemoembolization of liver cancer. Acad Radiol, 2018

18. Zheng J, Li J, Cui X et al (2013) Comparison of diagnostic sensitivity of Carm CT, DSA and CT in detecting small HCC. Hepatogastroenterology 60: 1509-1512

19. Kakeda S, Korogi Y, Ohnari N et al (2007) Usefulness of cone-beam volume CT with flat panel detectors in conjunction with catheter angiography for transcatheter arterial embolization. J Vasc Interv-Radiol 18:1508-1516

20. Wang C, Wang Q, Chen W et al (2015) DYNA CT arteriographic evaluation of hepatocellular carcinoma for treatment by trans-catheter arterial chemoembolization. Int J Clin Exp Med 8:20548-20555

21. Lee IJ, Chung JW, Yin YH et al (2015) Cone-beam computed tomography (CBCT) hepatic arteriography in chemoembolization for hepatocellular carcinoma: performance depicting tumors and tumor feeders. CardiovascIntervent Radiol 38:1218-1230

22. Pung L, Ahmad M, Mueller K et al (2017) The role of cone-beam CT in transcatheter arterial chemoembolization for hepatocellular carcinoma: a systematic review and meta-analysis. J Vasc Interv Radiol 28:334-341
23. Miyayama S, Yamashiro M, Hashimoto M et al (2013) Identification of small hepatocellular carcinoma and tumor-feeding branches with cone-beam CT guidance technology during trans catheter arterial chemoembolization. J Vasc Interv Radiol 24:501-508

24. Ushijima Y, Tajima T, Nishie A et al (2016) Detecting hepatic nodules and identifying feeding arteries of hepatocellular carcinoma: efficacy of conebeam computed tomography in transcatheter arterial chemoembolization. Hepatoma Res 2:231-236

25. Minami Y, Yagyu Y, Murakami T, Kudo M (2014) Tracking navigation imaging of transcatheter arterial chemoembolization for hepatocellular carcinoma using three-dimensional cone-beam CT angiography. Liver Cancer 3:53-61

26. Lee IJ, Chung JW, Yin YH et al (2014) Cone-beam CT hepatic arteriography in chemoembolization for hepatocellular carcinoma: angiographic image quality and its determining factors. J Vasc Interv Radiol 25(9):1369-1379

\section{Publisher's Note}

Springer Nature remains neutral with regard to jurisdictional claims in published maps and institutional affiliations.

\section{Submit your manuscript to a SpringerOpen ${ }^{\circ}$ journal and benefit from:}

- Convenient online submission

- Rigorous peer review

- Open access: articles freely available online

High visibility within the field

- Retaining the copyright to your article

Submit your next manuscript at $>$ springeropen.com 